DOI 10. 18307/2018. 0121

(C) 2018 by Journal of Lake Sciences

\title{
呼伦湖流域氢氧稳定同位素特征及其对水体蒸发的指示作用
}

\author{
高宏斌, 李畅游**, 孙标, 史小红, 赵胜男, 焚才睿 \\ (内蒙古农业大学水利与土木建筑工程学院, 呼和浩特 010018)
}

\begin{abstract}
摘 要: 以呼伦湖流域为例研究该区域氢氧稳定同位素在不同水体中的分布特征,并探讨氢氧稳定同位素对在该区域水 文过程的指示作用. 流域湖水、入湖河水、周边地下水水样的氢氧稳定同位素分析结果表明,夏季 8 月份湖水中的重氢氧 稳定同位素比 7 月份的更加富集. 而河水中氢氧稳定同位素在同一时间内的河流沿程上存在明显的差异,下游水体中的 氢氧稳定同位素要比上游更加富集. 研究区的河水和湖水的 $\delta^{18} \mathrm{O}-\delta \mathrm{D}$ 关系特征显示, 河水和湖水的 $\delta{ }^{18} \mathrm{O}-\delta \mathrm{D}$ 的关系点 全部位于当地降水线的右下方, 说明流域河水和湖水水体受到明显的蒸发作用. 而井水的 $\delta^{18} \mathrm{O}-\delta \mathrm{D}$ 的关系点大都靠近 当地大气降水线,说明这一区域的地下水主要是大气降水渗人地下形成. 利用氢氧稳定同位素分馏过程中的氢氧稳定同 位素的比率与剩余水体的关系, 并在考虑湿度因子的动力分馏模拟下, 计算出河水的剩余水体比例在 $0.85 \sim 0.96$ 之间, 而 湖水的剩余水体比例在 $0.71 \sim 0.77$ 之间. 最后, 利用氢氧稳定同位素质量平衡法对呼伦湖多年平均蒸发量进行了估算, 估 算的湖泊蒸发量结果与实测值相近, 相对误差为 $5.4 \%$, 说明方法可靠. 氢氧稳定同位素对于研究区域水文过程有着重要 的作用,在今后呼伦湖流域水文研究中有着更加广泛的应用空间.
\end{abstract}

关键词: 氢氧稳定同位素;分馏;蒸发;地下水;水文过程;呼伦湖

\section{Characteristics of hydrogen and oxygen stable isotopes in Lake Hulun Basin and its indic- ative function in evaporation}

\author{
GAO Hongbin, LI Changyou **, SUN Biao, SHI Xiaohong, ZHAO Shengnan \& FAN Cairui \\ (College of Water Conservancy and Civil Engineering, Inner Mongolia Agricultural University, Hohhot 010018, P.R.China)
}

\begin{abstract}
The aim of this paper is to analyze water stable isotopes $\left(\delta \mathrm{D}\right.$ and $\left.\delta^{18} \mathrm{O}\right)$ in Lake Hulun Basin, and to explore the indicative function of stable isotopes in hydrological cycle in this region. The analytical results of lake water, river water and well water around Lake Hulun indicate that there are temporal variations of stable isotopes in lake and river water and spatial variations in river water along the flow path. Furthermore, the relationship between $\delta^{18} \mathrm{O}$ and $\delta \mathrm{D}$ in lake water, river water and groundwater in Lake Hulun Basin shows the crossing points of lake and river water are located in the lower right of local meteoric water line, suggesting lake water and river water have suffered from evaporation. However, the crossing points of well water are located closely to the local meteoric water line except southwestern area (W1) around the lake, indicating all groundwater except W1 reflects a precipitation origin, and the weird high isotope values in W1 may have connections to the lake water and well water. Using the relationship between stable isotopic ratio in residual water and residual water proportion with consideration of the effect of temperature and humidity, the proportions of modern lake water and river water after evaporation are calculated as $0.85-0.96$ for river water, and 0.710.77 for lake water. Obviously, the extent of lake water evaporation is greater than that of river water, with $4 \%-15 \%$ water $l o s s$ for rivers and $23 \%-29 \%$ water loss for the lake. In addition, the annual lake water evaporation was calculated based on isotopic mass balance method, the low relative error $(5.4 \%)$ compared with measured data indicates this model is appropriate to calculate water evaporation for Lake Hulun. Using stable isotope $\left(\delta \mathrm{D}\right.$ and $\left.\delta^{18} \mathrm{O}\right)$ is an essential method to study on hydrological cycle in a basin, thus stable isotope still has a wide application space in hydrological cycle in Lake Hulun Basin.
\end{abstract}

Keywords: Hydrogen and oxygen stable isotopes; fractionation; evaporation; underground water; hydrological process; Lake Hulun

* 国家自然科学基金项目 $(51339002,51409288,51509133,51569019)$ 、内蒙古农业大学优秀青年科学基金项目 (2014XYQ-10) 和国家留学基金委项目 (201408150013) 联合资助. 2016-11-25 收稿; 2017 - 01 - 13 收修改稿. 高宏斌(1988 ), 男, 博士研究生; E-mail: gaohongbin0922@126.com.

** 通信作者; E-mail: nndlichangyou@163.com. 
地球上的水分通过降水、蒸散发、下渗、径流等过程控制着水分的循环. 自然界中蒸发和凝结作用是造 成水体中的氢氧稳定同位素分馏的重要方式, 也是造成各种地表水体的氢氧稳定同位素组成差别并且具有 一定分布规律的重要原因 ${ }^{[1]}$. 一方面, 水分子在蒸发、凝结过程中发生分馏, 造成组成水分子的氢氧稳定同 位素含量发生变化,使得不同状态的水体含有不同的氢氧稳定同位素值 ${ }^{[2-3]}$. 利用这种异质性,氢氧稳定同 位素作为示踪剂成为近年来指示流域水循环、确定地表水和地下径流的来源和地表水、地下水相互作用关 系等研究方面的重要技术手段. 例如, Clark 等 ${ }^{[4]}$ 利用氢氧稳定同位素的示踪性, 明确了不同时间、空间尺度 下的地下水水文循环过程; Wassenaar 等 ${ }^{[5]}$ 和 Wang 等 ${ }^{[6]}$ 运用氢氧稳定同位素对地下水进行了研究, 识别出 了地下水的补给源和补给区域; Currell 等 ${ }^{[7]}$ 和 Cui 等 ${ }^{[8]}$ 对湖泊和地下水的氢氧稳定同位素进行了研究,明 确了湖水与地下水的混合区域和补给关系. 另一方面, 通过对水体蒸发过程中氢氧稳定同位素的分馏机制 的研究, 建立水体蒸发分馏模型模拟氢氧稳定同位素的分馏规律, 以及确定水体蒸发量是氢氧稳定同位素 在水文循环研究中的另一个应用. 例如, 田立德等 ${ }^{[9]}$ 在 1998 年首次对青藏高原中部地区进行了水体氢氧稳 定同位素在蒸发过程中的研究, 通过野外水蒸发实验和模拟计算结合, 认为水在蒸发过程中其剩余水体中的 氧氢稳定同位素值与剩余水体显著相关, 而且蒸发分馏因子的大小受相对湿度的影响. 马斌等 ${ }^{[10]}$ 对华北平原 水蒸发过程中氢氧稳定同位素的分馏特征进行了研究, 获得了氢氧稳定同位素变化量与累积蒸发量的关系, 并且认为盐分对蒸发分馏产生的影响较低. 钱会等 ${ }^{[11]}$ 使用瑞利平衡分馏方程对河水的蒸发比例进行了计算, 表明河水的累计蒸发比例相对最初的水量可达到 20\% 48\%. 肖薇等 ${ }^{[12-14]}$ 利用氢氧稳定同位素的比率估算了 湖泊的多年平均蒸发量,并与实测蒸发量比较,认为利用氢氧稳定同位素计算湖泊蒸发量的方法可靠.

鉴于上述, 氢氧稳定同位素对于研究区域水文过程有着重要的作用. 目前对呼伦湖流域水文过程中的 氢氧稳定同位素特征的相关研究鲜有报道, 一定程度上制约了对该流域的水文循环过程、地表水与地下水 相互关系等方面的深刻认识. 本文基于对内蒙古呼伦湖流域不同水体的氢氧稳定同位素组成分析, 利用氢 氧稳定同位素的指示作用对呼伦湖流域水文过程的机理进行研究, 为流域水资源持续利用和生态环境建设

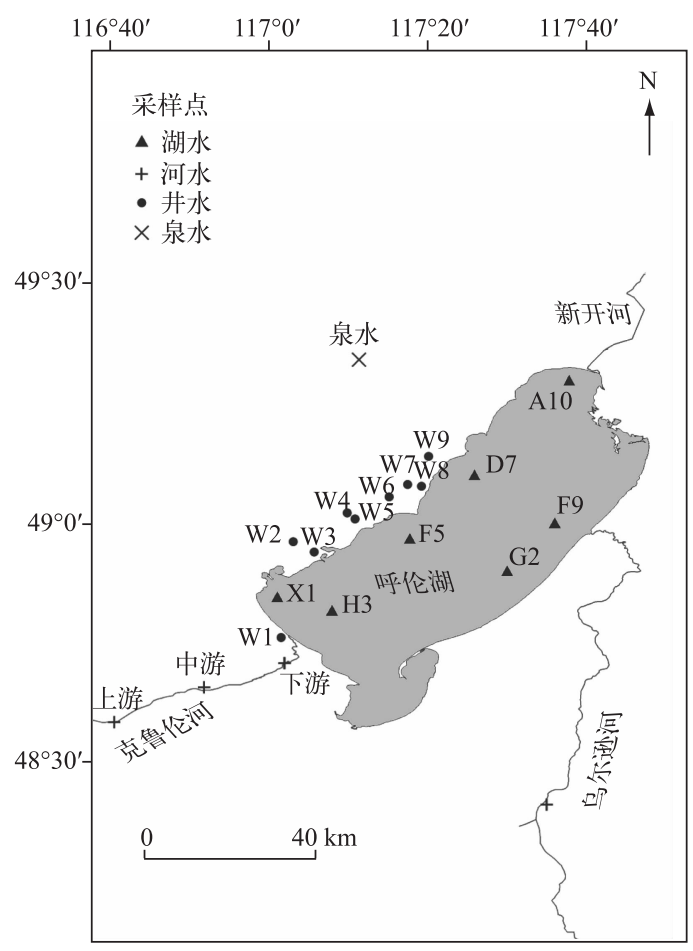

图 1 研究区及采样点位置

Fig. 1 Location of the study area and sampling sites
提供科学参考.

\section{1 材料与方法}

\section{1 研究区概况}

呼伦湖 $\left(48^{\circ} 33^{\prime} \sim 49^{\circ} 20^{\prime} \mathrm{N}, 116^{\circ} 58^{\prime} \sim 117^{\circ} 48^{\prime} \mathrm{E}\right)$ 为中国第五大湖, 也是我国北方最大的湖泊, 位于内 蒙古自治区满洲里市及新巴尔虎左旗、新巴尔虎右 旗之间 (图 1). 呼伦湖全流域面积为 25.6 万 $\mathrm{km}^{2}$, 我 国部分为 9.3 万 $\mathrm{km}^{2}$, 所占比例为 $37.3 \%$; 蒙古国部分 为 16.3 万 $\mathrm{km}^{2}$, 所占比例达 $63.7 \%{ }^{[15]}$. 呼伦湖地区处 在半干旱的高纬度地带, 属中温带大陆性气候, 年平均 气温为 $1.2^{\circ} \mathrm{C}$, 年平均降雨量为 $235 \mathrm{~mm}$, 年平均蒸发量 为 $1470 \mathrm{~mm}$ 左右, 最大湿度达到 $80 \%$ (来自 $1961-$ 2013 年水文站数据). 呼伦湖湖水的补给除了来源大 气降水外, 主要来源于蒙古境内肯特山脉的克鲁伦河, 以及连接贝尔湖的乌尔逊河. 其中每年接纳平均降水 量大约为 6.29 亿 $\mathrm{m}^{3}$; 多年平均径流量为 12.58 亿 $\mathrm{m}^{3}$; 除此之外, 呼伦湖区域的地下水也是湖水重要的补给 来源, 年补给量约为 3.90 亿 $\mathrm{m}^{3[16]}$. 呼伦湖近年来由于 其出流河口被人为控制, 基本上成了内陆封闭湖泊, 因 此湖水除了之前个别年份通过新开河泄水外, 其水量 损失主要是来自湖面的蒸发, 多年平均水面蒸发量为 $1103 \mathrm{~mm}$, 折算成水量为 25.80 亿 $\mathrm{m}^{3[16]}$. 近年来, 由于 
气候和人为因素的影响, 呼伦湖径流量减少, 湖泊水位下降, 面积减小, 水质变差, 同时, 由于水土流失和草地 退化使得进人呼伦湖的污染物浓度升高 ${ }^{[16]}$, 湖泊水环境及其生态环境的恶化受到了广泛的关注.

\section{2 样品的采集与测定分析}

根据呼伦湖的水系特征及水文地质条件,于 2014 年夏季的 6-8 月对呼伦湖湖面、两条人湖河流 (克鲁 伦河、乌尔逊河)、周边地下井水 (井深在 1 15 m 之间)、以及泉水分别采样, 共采集 31 个水样. 其中 6 月 26 日分别采集克鲁伦河的上、中、下游 3 个水样; 7 月 4 日和 8 月 3 日分别采集呼伦湖湖面水样,每次 7 个, 共 14 个, 以及克鲁伦河下游处和乌尔逊河相同位置处各 2 个,共 4 个; 7 月 30 日在湖周边的西南部区域采集 9 个地下井水样品和 1 个泉水样. 采样过程中用 GPS 定位采样点 (图 1), 采集的所有水样立即用封口膜密封 在 $100 \mathrm{ml}$ 的聚乙烯瓶中,放于保温箱,带回实验室.

所有水样 (湖水、井水、河水、泉水) 在测定分析前都经 $0.45 \mu \mathrm{m}$ 的滤膜过滤. 氢氧稳定同位素采用内蒙 古农业大学水环境实验室的高精度的美国 LGR 公司的液态水氢氧同位素分析仪测定 (仪器型号: LMIA-V2 (DLT-100), 精度 $\delta \mathrm{D}: 1 \% 0, \delta^{18} 0: 0.1 \%$ ). 计算结果以维也纳平均海水 VSMOW 为标准, 公式为:

$$
\delta[\%]=\left[R_{\text {sample }} / R_{\text {standard }}-1\right] \times 1000
$$

式中, $\delta$ 为同位素组成, $R$ 为重氢氧稳定同位素与轻氢氧稳定同位素的比值.

\section{2 结果与分析}

\section{1 呼伦湖流域不同水体氢氧稳定同位素的分布特征}

呼伦湖流域不同水体具有不同的氢氧稳定同位素组成特征 (表 1). 其中, 湖水中的氢氧稳定同位素的 值最大, $\delta \mathrm{D}$ 分布在 $-72.83 \% 0 \sim-63.44 \%$ 之间, $\delta^{18} \mathrm{O}$ 分布在 $-8.55 \%$ - 7.12\%0 之间, 河水的氢氧稳定同位素值 较湖水的次之, $\delta \mathrm{D}$ 分布在 $-95.99 \%$ $-80.69 \%$ 之间, $\delta^{18} \mathrm{O}$ 分布在 $-12.35 \% 0 \sim-10.17 \%$ 之间, 地下水除了 $\mathrm{W} 1$ 点 $\left(\delta \mathrm{D}:-78.52 \%, \delta^{18} \mathrm{O}:-8.91 \% 0\right)$ 和 $\mathrm{W} 3$ 点 $\left(\delta \mathrm{D}:-72.02 \% 0, \delta^{18} \mathrm{O}:-9.41 \% 0\right)$ 异常偏正外, 其他的井水和泉水的 氢氧稳定同位素值都接近或小于附近河水中的值, $\delta \mathrm{D}$ 分布在 $-120.87 \% 0 \sim-86.86 \%$ 之间, $\delta^{18} 0$ 分布在 $-15.91 \%$ - $11.40 \%$ 之间.

\section{表 1 呼伦湖流域各种水体中氢氧 稳定同位素分析结果 *}

Tab.1 The results of $\delta \mathrm{D}$ and $\delta^{18} \mathrm{O}$ in water samples from Lake Hulun Basin

\begin{tabular}{|c|c|c|c|c|c|c|c|}
\hline 水体 & 样品号(日期) & ${ }^{18} \mathrm{O}_{\mathrm{V} \text {-smow }} / \%$ & $\mathrm{D}_{\text {V-sMow }} / \%$ & 水体 & 样品号(日期) & ${ }^{18} \mathrm{O}_{\mathrm{V} \text {-suow }} / \%$ & $\mathrm{D}_{\mathrm{V} \text {-SMow }} / \%$ \\
\hline \multirow[t]{14}{*}{ 湖水 } & $\mathrm{A} 10$ ( 7 月 4 日) & -8.55 & -72.83 & 河水 & KLL下 $(6$ 月 26 日) & -10.98 & -85.72 \\
\hline & D7( 7 月 4 日) & -7.83 & -66.59 & & KLL 下 ( 7 月 4 日) & -10.46 & -84.00 \\
\hline & F5( 7 月 4 日) & -7.61 & -66.36 & & KLL 下 ( 8 月 3 日) & -10.30 & -83.20 \\
\hline & $\mathrm{F} 9$ ( 7 月 4 日) & -7.66 & -65.71 & & $\mathrm{WEX}$ (7月 4 日) & -11.24 & -87.56 \\
\hline & $\mathrm{G} 2$ ( 7 月 4 日) & -7.47 & -65.70 & & $\operatorname{WEX}(8$ 月 3 日) & -10.17 & -80.69 \\
\hline & $\mathrm{H} 3$ ( 7 月 4 日) & -7.33 & -65.71 & 地下水 & $\mathrm{W} 1$ ( 7 月 30 日) & -8.91 & -78.52 \\
\hline & X1( 7 月 4 日) & -7.49 & -65.75 & & $\mathrm{~W} 2$ ( 7 月 30 日) & -11.40 & -86.86 \\
\hline & $\mathrm{A} 10$ ( 8 月 3 日) & -8.21 & -71.87 & & $\mathrm{~W} 3$ ( 7 月 30 日) & -9.41 & -72.02 \\
\hline & D7 ( 8 月 3 日) & -7.38 & -67.24 & & $\mathrm{~W} 4$ ( 7 月 30 日) & -11.98 & -89.61 \\
\hline & $\mathrm{F} 5$ ( 8 月 3 日) & -7.12 & -65.91 & & $\mathrm{~W} 5$ ( 7 月 30 日) & -12.33 & -93.05 \\
\hline & $\mathrm{F} 9$ ( 8 月 3 日) & -7.16 & -65.88 & & $\mathrm{~W} 6(7$ 月 30 日) & -13.34 & -100.54 \\
\hline & $\mathrm{G} 2$ ( 8 月 3 日) & -7.27 & -65.21 & & $\mathrm{~W} 7$ ( 7 月 30 日) & -11.64 & -89.38 \\
\hline & H3 ( 8 月 3 日) & -7.25 & -65.23 & & W8( 7 月 30 日) & -15.91 & -120.87 \\
\hline & X1(8月 3 日) & -7.12 & -63.44 & & W9(7月 30 日) & -14.25 & -105.24 \\
\hline \multirow[t]{2}{*}{ 河水 } & $\mathrm{KLL}$ 上 $(6$ 月 26 日) & -12.35 & -95.99 & & 泉水 $(7$ 月 30 日) & -13.06 & -98.35 \\
\hline & $\mathrm{KLL}$ 中 $(6$ 月 26 日) & -11.84 & -94.48 & & & & \\
\hline
\end{tabular}

* KLL 表示克鲁伦河, WEX 表示乌尔逊河, W 表示井水. 


\section{2 湖水和河水的氢氧稳定同位素组成随时间的变化}

氢氧稳定同位素组成的季节变化明显,同时在同一季节由于不同时间段的温度对水体蒸发作用的影响 程度不同, 因此氢氧稳定同位素组成也随之变化. 比较夏季两次不同时间获得的样品的氢氧稳定同位素分 析结果 (图 2), 总体上看, 除了湖面上 D7 点和 $\mathrm{F} 9$ 点 8 月样品的 $8 \mathrm{D}$ 值小于 7 月, 其他的点位 8 月样品的 $8 \mathrm{D}$ 和 $\delta^{18} \mathrm{O}$ 值要高于 7 月样品的, 特别是 $\delta^{18} \mathrm{O}$ 值在不同时间上的体现更为明显, 说明呼伦湖在 7 月到 8 月之间 经过强烈的蒸发作用, 湖水中的重氢氧稳定同位素更加富集,一定程度上体现了该地区水体中氢氧稳定同 位素组成在夏季随时间的变化. 而 $\delta^{18} \mathrm{O}$ 较 $\delta \mathrm{D}$ 变化更为明显, 可能是由于在通常水体中氧稳定同位素的含 量要高于氢稳定同位素, 测量时误差小而结果更加准确, 也正因为此, 很多研究中氧稳定同位素的应用要比 氢稳定同位素广泛一些.
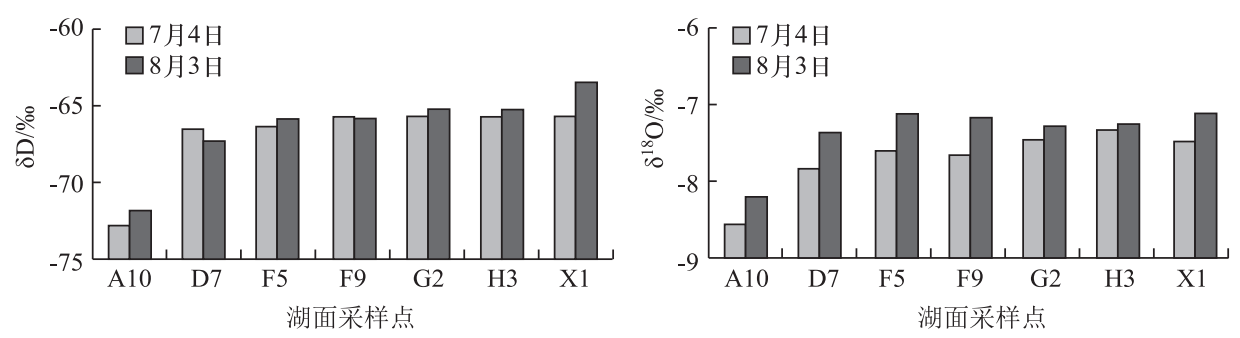

图 2 呼伦湖各采样点水体中氢氧稳定同位素组成随时间的变化

Fig. 2 Temporal variations of $\delta \mathrm{D}$ and $\delta^{18} \mathrm{O}$ of each sampling site in Lake Hulun

在野外实验过程中由于交通原因, 对克鲁伦河的取样更为密集, 因此将克鲁伦河同一位置下游处 3 个 不同时间段的水样氢氧稳定同位素作比较 (图 3), 发现河水的 $\delta \mathrm{D}$ 和 $\delta^{18} \mathrm{O}$ 随时间的变化与湖水具有相似的 特征, 从 6 月到 8 月初, $\delta \mathrm{D}$ 和 $\delta^{18} \mathrm{O}$ 表现为明显的递增趋势, 同样反映了蒸发作用对河水中氢氧稳定同位素 分布特征的影响.
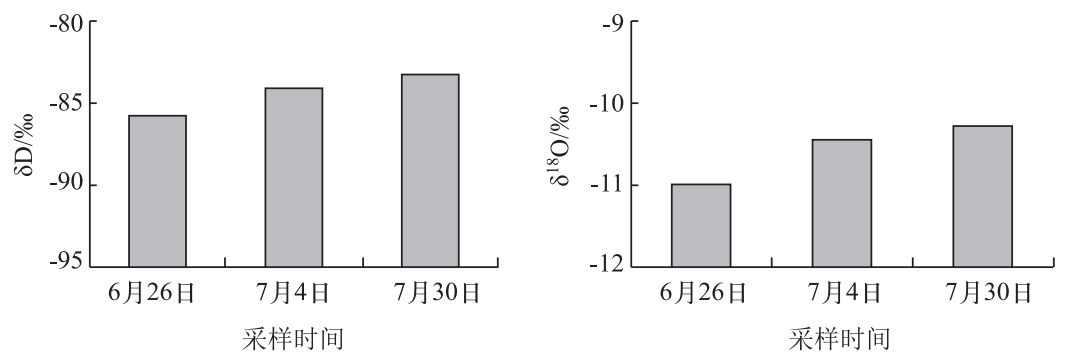

图 3 克鲁伦河水体中氢氧稳定同位素组成随时间的变化

Fig. 3 Temporal variations of $\delta \mathrm{D}$ and $\delta^{18} \mathrm{O}$ of water samples in Kelulun River

\section{3 河水氢氧稳定同位素组成的沿程变化}

克鲁伦河水体中的 $\delta \mathrm{D}$ 和 $\delta^{18} \mathrm{O}$ 从上游到下游呈明显增大的趋势, $\delta \mathrm{D}$ 从 $-95.99 \%$ 增大到 $-85.72 \%, \delta^{18} \mathrm{O}$ 从-12.35\% 增大到-10.98\%o (图 4). 克鲁伦河氢氧稳定同位素的这种沿程分布变化,一方面与上、中、下游汇 人水体的氢氧稳定同位素特征有关, 另外主要还是与河流流动过程中沿程受到的蒸发作用有关.

\section{3 讨论}

\section{1 呼伦湖流域大气降水和其他水体中 $\delta^{18} \mathrm{O}-\delta \mathrm{D}$ 的关系特征}

研究流域氢氧稳定同位素的分布特征, 确定当地降水线是研究的基础. Craig ${ }^{\left[{ }^{[17]}\right.}$ 在研究北美大气降水时 发现大气降水的氢氧稳定同位素组成呈线性变化,并确定了最初的全球大气降水方程. 但后来研究者发现 由于不同地区海拔、地貌、气候等条件的不同, 各地大气降水线偏离全球降水线的情况各不相同, 因此为了 

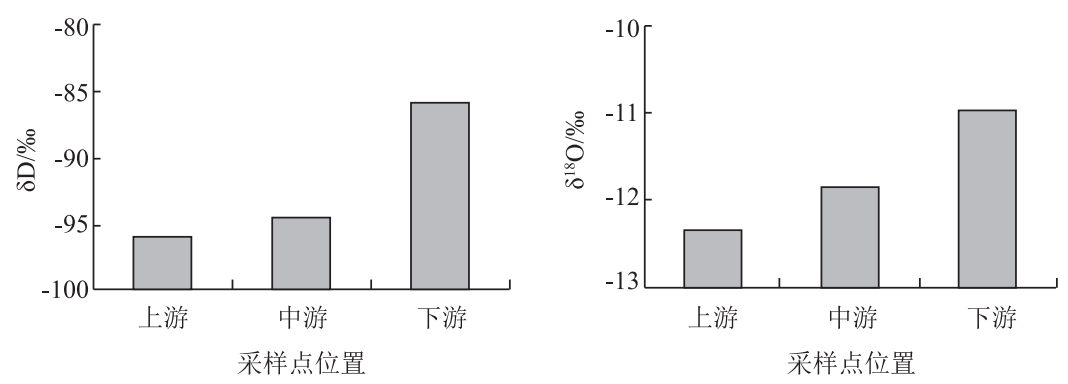

图 4 克鲁伦河水体中氢氧稳定同位素组成的沿程变化

Fig.4 Variations of $\delta D$ and $\delta^{18} \mathrm{O}$ of water samples in Kelulun River from upstream to downstream

更加精准合理地研究当地的氢氧稳定同位素特征, 应建立相应的当地降水线. 目前, 在呼伦湖流域氢氧稳定 同位素的研究还鲜有报道, 同时受研究区流域降水资料的限制, 当地大气降水线不能利用该区域实测的数 据生成. Tsujimura 等 ${ }^{[18]}$ 在 2007 年对克鲁伦河流域的不同水域同位素进行了研究,并确定了该区域的大气 降水线, 由于文献中的研究区克鲁伦河流域属于呼伦湖流域的一个部分, 二者的地理位置和气候环境相差 不大,因此本文采用该结果作为呼伦湖流域的当地大气降水线,关系式如下：

$$
\delta \mathrm{D}=7.5 \delta^{18} \mathrm{O}+2.1
$$

一个地区水体蒸发线反映了区域气象参数, 不 同气候条件下的水体的蒸发线的斜率会有所区别. 结合本研究获得的数据,得出呼伦湖流域不同水体 $\delta^{18} \mathrm{O}-\delta \mathrm{D}$ 的关系 (图 5), 研究区的河水和湖水 $\delta^{18} \mathrm{O}-\delta \mathrm{D}$ 的关系点全部位于当地降水线的右下 方, 说明流域河水和湖水水体受到明显的蒸发作 用. 而湖水的 $\delta^{18} \mathrm{O}-\delta \mathrm{D}$ 关系点分布范围沿蒸发线 并位于最上方,河水的分布范围位于下方, 说明湖 水受到的蒸发作用更大. 研究区井水和泉水的 $\delta^{18} \mathrm{O}-\delta \mathrm{D}$ 关系点除了 $\mathrm{W} 1$ 外, 全部落在当地降水线 附近, 说明这一区域的地下水主要是大气降水渗人 地下形成,这种地下水氢氧稳定同位素特征是一种 普遍的现象, 在其他研究中也多有报道 ${ }^{[19]}$. W 1 点

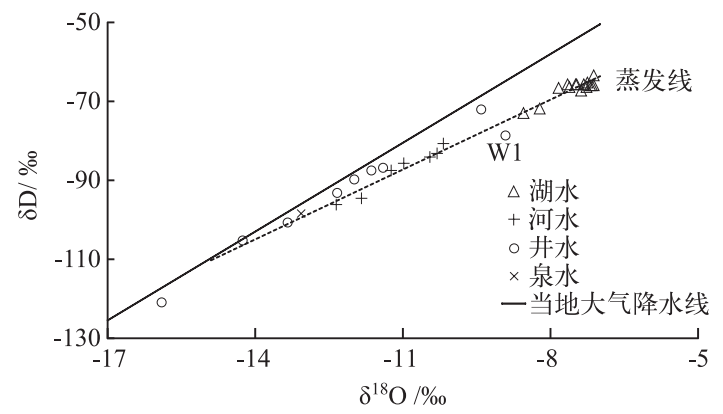

图 5 呼伦湖湖水、河水、井水和泉水 $\delta^{18} \mathrm{O}-\delta \mathrm{D}$ 的关系

Fig.5 The cross-plot of $\delta^{18} \mathrm{O}$ and $\delta \mathrm{D}$ from water of Lake Hulun, river water, well water and spring 井水的 $\delta^{18} \mathrm{O}-\delta \mathrm{D}$ 关系点偏离至当地降水线的右下 方, 说明该井水体也受到明显的蒸发作用. 由于井水位于地下不易受到强烈的蒸发作用,且该井水 $\delta^{18} \mathrm{O}-\delta \mathrm{D}$ 关系点介于湖水之下和大部分地下水之上, 因此可能是因为蒸发的湖水进人该井水并与蒸发作用不明显的 地下水发生了混合作用.

\section{2 剩余水体中氢氧稳定同位素组成与剩余水体量比例的关系}

蒸发在水文循环中是重要的环节, 特别是对于半干旱区的呼伦湖流域, 河水和湖水的蒸发是水量流失 的主要过程. 水体蒸发过程中,相对轻的氢氧同位素水分子间的氢键优先被破坏,使部分轻的氢氧同位素先 形成蒸汽离开液面, 重的氢氧同位素逐渐被富集在剩余水体中, 这一过程称为分馏 ${ }^{[3,9]}$. 在瑞利平衡蒸发条 件下,剩余水体的氢氧稳定同位素组成 $(\delta)$ 与受蒸发后剩余水体和原始水体的比率 $(f)$ 呈指数关系, 服从瑞 利蒸发分馏模型 ${ }^{[20]}$ :

$$
\delta=\left(\delta_{0}+1\right) f^{(a-1)}-1
$$

式中, $\delta_{0}$ 为水体氢氧稳定同位素的初始组成, $f$ 为剩余水体的比例, $a$ 为分馏系数. 由氢氧稳定同位素确定的 当地降水线和蒸发线的交点常常被认为是受蒸发水体氢氧稳定同位素的最初组成, 故本文选用这一交点 $\left(\delta^{18} 0:-13.00 \%, \delta \mathrm{D}:-99.97 \% 0\right)$ 作为 $\delta_{0}$ 值, $\delta$ 为呼伦湖河水和湖水的实测值, 因此, 只要确定分馏系数 $a$, 就 
可以计算出剩余水体量的比例. 由于式 (3) 中 $\delta$ 的绝对值很小,为方便起见,该式可简化为:

$$
\delta-\delta_{0}=10^{3}(a-1) \ln f
$$

在瑞利平衡分馏过程中, 温度是控制氢氧稳定同位素分馏的主要因素, 分馏系数随环境温度的变化而 改变. 由 Majoube ${ }^{[21]}$ 提出的温度从 $273.15 \mathrm{~K}$ 到 $373.15 \mathrm{~K}$ 变化时的经验方程, 蒸发分馏系数 $a$ 与温度 $(T)$ 存 在以下关系:

$$
\begin{aligned}
& 10^{3} \ln a \mathrm{O}^{18}=1.137\left(\frac{10^{6}}{T^{2}}\right)-0.4156\left(\frac{10^{3}}{T}\right)-2.0667 \\
& 10^{3} \ln a \mathrm{D}=24.844\left(\frac{10^{6}}{T^{2}}\right)-76.248\left(\frac{10^{3}}{T}\right)+51.612
\end{aligned}
$$

因此, 可以根据上式计算出不同温度下的分馏系数. 结果表明, 氢氧稳定同位素的分馏系数与温度呈反

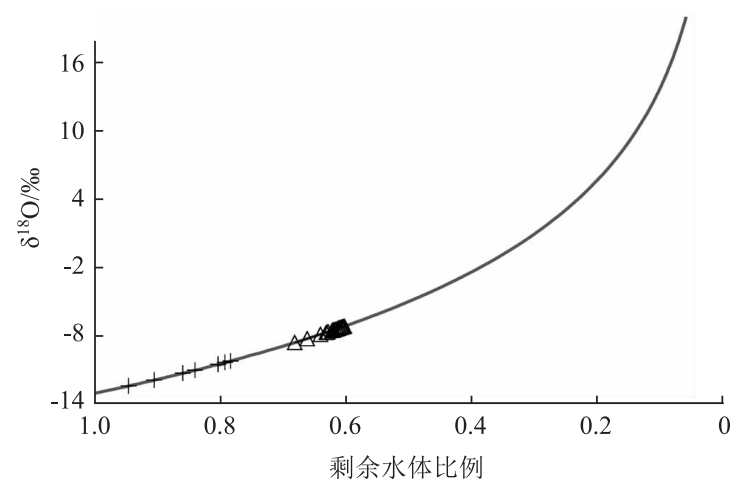

图 6 平衡条件下剩余湖水、河水中 $\delta^{18} \mathrm{O}$

与剩余水体比例的关系 $\left(T=1.2^{\circ} \mathrm{C}\right)$

Fig.6 Relationship between $\delta^{18} \mathrm{O}$ and $f$ in residual lake water and river water under equilibrium condition $\left(T=1.2^{\circ} \mathrm{C}\right)$
比, 温度越低, 分馏系数越大, 分馏程度就越深.

根据呼伦湖流域的气象数据得知, 呼伦湖常 年平均温度 $1.2^{\circ} \mathrm{C}$. 因此, 采用 $T=1.2^{\circ} \mathrm{C}$ 计算出的 蒸馏系数 $a=1.0116$ 作为确定呼伦湖湖水和河水 剩余水体的比例. 根据前人的研究发现, 计算水体 蒸发量时使用 $\delta^{18} \mathrm{O}$ 更为可靠 ${ }^{[11]}$, 故本文采用 $\delta^{18} \mathrm{O}$ 的计算结果 (图 6). 如图 6 所示, 通过呼伦湖流域 水体 $\delta^{18} \mathrm{O}$ 的实测值落在 $\delta^{18} \mathrm{O}$ 与剩余水体比例的 模拟线上的位置, 可以清楚地确定水体受到的蒸 发程度. 河水和湖水由于受蒸发程度的不同, 剩余 水体比率的分布分为两个部分, 河水的剩余水体 比例在 $0.78 \sim 0.95$ 之间, 而湖水的剩余水体比例 在 $0.60 \sim 0.68$ 之间, 说明现有的河水由于蒸发损 失的量在最初水体的 5\% 22\% 之间, 而湖水由于 封闭状态下受到的累积蒸发更大, 损失量在最初 水体的 32\% 40\% 之间.

已有大量研究表明, 由于分子扩散作用所引起的动力效应和水体与空气氢氧同位素之间的相互关系和 交换因子等的影响, 自然条件下的实际蒸发分馏与理想的平衡蒸发分馏存在一定的差异, 这主要是因为实 际水的蒸发不仅有平衡蒸馏过程, 还有动力蒸馏的过程 ${ }^{[22-23]}$. 动力蒸馏主要与大气湿度有关, Gonfiantini ${ }^{[24]}$ 给出分馏系数与湿度的计算关系式:

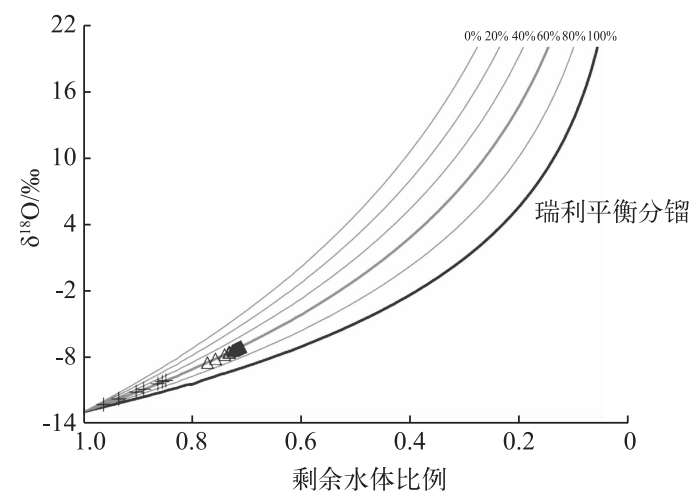

图 7 不同相对湿度下剩余水体中 $\delta^{18} \mathrm{O}$ 与剩余水体比例的关系 $\left(T=1.2^{\circ} \mathrm{C}\right)$

Fig.7 Relationship between $\delta^{18} \mathrm{O}$ and $f$ in residual water under different relative humidities $\left(T=1.2^{\circ} \mathrm{C}\right)$

$$
\Delta \varepsilon^{18} \mathrm{O}=14.2(1-h) \times 10^{-3}
$$

式中, $\varepsilon$ 为富集系数, $\varepsilon=1000(1-a) ; h$ 为湖面的相 对湿度. 据此可以模拟出不同大气相对湿度下的水 中 $\delta^{18} \mathrm{O}$ 的分馏过程, 并与在相同温度下 $(T=$ $\left.1.2^{\circ} \mathrm{C}\right)$ 的瑞利平衡分馏过程作对比. 结果表明, 湿 度对蒸发分馏过程有着重要的影响, 随着湿度的增 加,剩余水体中 $\delta^{18} \mathrm{O}$ 随剩余水体比例的变化逐渐 减小 (图 7). 对比瑞利平衡分馏 $(h=100 \%$ ), 其他 相对湿度下剩余水体中 $\delta^{18} 0$ 的分馏程度都要大于 瑞利平衡分馏, 这再次说明了在实际水体蒸发过程 中, 由湿度因子引起的动力分馏作用必须予以 考虑.

根据呼伦湖实测气象数据得知, 多年平均相对 湿度大约在 $60 \%$ 左右, 利用该湿度值计算出的蒸发 
分馏过程中剩余水体中 $\delta^{18} O$ 随剩余水体比例模拟线 (灰色粗线) 以及相应的实测水体中 $\delta^{18} O$ 的值如图 7 所 示, 根据实测 $\delta^{18} O$ 值落在模拟线上的位置, 可以看出计算出的河水和湖水水体剩余水体比例较瑞利平衡蒸 馏下的大很多, 河水的剩余水体比例在 $0.85 \sim 0.96$ 之间, 而湖水的剩余水体比例在 $0.71 \sim 0.77$ 之间, 对应的 河水蒸发损失的量占最初水体的 $4 \% \sim 15 \%$, 而湖水损失量占最初水体的 $23 \% \sim 29 \%$.

\section{3 氢氧稳定同位素平衡法估算湖泊蒸发量}

氢氧稳定同位素方法对于推求湖泊蒸发量是一个非常有效的工具,特别是对于实测资料缺乏的研究区. 通常使用水量平衡方法计算蒸发量时, 由于无法准确地确定水量平衡各项的要素, 尤其是地下水人湖量, 造 成计算结果的不确定性. 利用氢氧稳定同位素计算湖泊蒸发量的优势在于不必测定人湖水量, 只需测定各 种人湖水体的氢氧稳定同位素组成, 结合水量平衡方程和氢氧稳定同位素物质平衡方程, 就可计算出湖泊 的蒸发量.

湖泊中的水量平衡方程可以表示为:

$$
\frac{\mathrm{d} V}{\mathrm{~d} t}=I+P-Q-E
$$

式中, $V$ 为湖泊的体积, $I$ 为进人湖泊的水量, $P$ 为降水量, $Q$ 为流出湖泊的水量, $E$ 为湖泊的蒸发量.

而对于一个浅水湖泊,由于其混合程度较好, 氢氧稳定同位素物质平衡方程可以表示为:

$$
\frac{\mathrm{d} \delta_{1} V}{\mathrm{~d} t}=\delta_{\mathrm{i}} \cdot I+\delta_{\mathrm{p}} \cdot P-\delta_{\mathrm{q}} \cdot Q-\delta_{\mathrm{e}} \cdot E
$$

式中, $\delta_{1} 、 \delta_{\mathrm{i}} 、 \delta_{\mathrm{p}} 、 \delta_{\mathrm{q}}$ 和 $\delta_{\mathrm{e}}$ 分别为湖水、人湖水、降水、出流水和湖泊蒸发水氢氧稳定同位素的组成. 由式 (8) 和 (9) 合并 (湖泊完全混合下, $\delta_{1}=\delta_{\mathrm{q}}$ ), 湖泊蒸发量 $E$ 可表示为:

$$
E=\frac{\frac{\mathrm{d} V}{\mathrm{~d} t}\left(\delta_{1}-\delta_{\mathrm{i}}\right)+V \frac{\mathrm{d} \delta_{1}}{\mathrm{~d} t}+P\left(\delta_{\mathrm{i}}-\delta_{\mathrm{p}}\right)-Q\left(\delta_{\mathrm{i}}-\delta_{1}\right)}{\delta_{\mathrm{i}}-\delta_{\mathrm{e}}}
$$

根据 Saxena ${ }^{[25]}$ 通过模型给出的湖水蒸发过程中蒸发水汽氢氧同位素组成 $\delta_{\mathrm{e}}$ 的求法:

$$
\delta_{\mathrm{e}}=\frac{\frac{1}{a}\left(1+\delta_{1}\right)-h\left(1+\delta_{\mathrm{a}}\right)}{(1-h) \beta}-1
$$

式中, $\delta_{\mathrm{a}}$ 为大气水汽为氢氧稳定同位素组成; $h=60 \% ; \beta$ 为重轻分子传输阻力系数, 大约为 1.013 ; 大气中氢氧 同位素的 $\delta_{\mathrm{a}}$ 按照下式计算 ${ }^{[26]}$ :

$$
\delta_{\mathrm{a}}=-0.014 T^{2}+0.67 T-23.4
$$

式中, $T$ 为湖面气温 $\left({ }^{\circ} \mathrm{C}\right)$, 采用呼伦湖气象数据的常年平均温度 $1.2^{\circ} \mathrm{C}$.

呼伦湖是一个受湖泊水位控制出流的湖泊,近年来由于水位较低成为了封闭性湖泊, 因此水面蒸发是 湖泊的唯一支出项, $Q$ 可以忽略不计. 假定湖水在短时间内的氢氧稳定同位素组成保持不变, 则有 $\mathrm{d} \delta_{1} / \mathrm{d} t=$ 0 ,式 (10) 可简化为:

$$
E=\frac{\frac{\mathrm{d} V}{\mathrm{~d} t}\left(\delta_{1}-\delta_{\mathrm{i}}\right)+P\left(\delta_{\mathrm{i}}-\delta_{\mathrm{p}}\right)}{\delta_{\mathrm{i}}-\delta_{\mathrm{e}}}
$$

式中, $\delta_{1}$ 取所有实测湖水样品中测得的 $\delta^{18} O$ 的算术平均值, 为 $-7.51 \%$; $\delta_{\mathrm{i}}$ 可通过当地降水线与蒸发线的交点 获得, 为 $-13.00 \%$, 这一方法已被证明可以很好地估算湖泊的人湖水中氢氧稳定同位素的权重值 ${ }^{[27-28]} . \delta_{\mathrm{p}}$ 来 自 IAEA (国际原子能机构) 设立在齐齐哈尔长期监测站 $\delta^{18} \mathrm{O}$ 的平均值, 取值为 $-15.00 \%$, 由公式 (12)、(13) 求得的 $\delta_{\mathrm{a}}$ 和 $\delta_{\mathrm{e}}$ 分别为 $-23.40 \%$ 和 $-13.75 \%$.

根据呼伦湖周边水文站 1961-2013 年的实测资料, 得知呼伦湖 50 多年来平均湖泊面积为 $2020 \mathrm{~km}^{2}$, 多 年蓄水量变化为 1.25 亿 $\mathrm{m}^{3}$, 可换算为 $61.9 \mathrm{~mm}$; 多年平均降雨量为 $235 \mathrm{~mm}$; 多年平均蒸发量为 $1470 \mathrm{~mm}$ (水 文站 $20 \mathrm{~cm}$ 蒸发血) 左右, 折合水面蒸发量为 $1140 \mathrm{~mm}$ (折算系数 0.776 来源于文献[29]). 将各项及参数代 人公式(14) 中, 可得出呼伦湖多年平均蒸发量为 $1078 \mathrm{~mm}$, 与实测值 1140 相差 $62 \mathrm{~mm}$, 相对误差为 $5.4 \%$, 这 
可能是在计算过程中由于涉及的参数较多出现的误差, 特别 $\delta_{\mathrm{e}}$ 值是按照经验公式计算得出, 计算值的偏大 或偏小都对蒸发量的估算有很大的影响. 另外,计算中采用不同水体中平均氢氧稳定同位素组成可能会因 为实测样品量的不足而存在一定的误差. 但整体来说利用氢氧稳定同位素平衡方法估算呼伦湖蒸发量是可 靠的.

\section{4 结论}

1) 对研究区不同水体中的氢氧稳定同位素进行研究, 结果表明湖水和河水中氢氧稳定同位素在时间和 空间上由于受不同程度蒸发作用的影响都存在明显的差异. 此外, 根据氢氧稳定同位素在不同水体中的 $\delta^{18} \mathrm{O}-\delta \mathrm{D}$ 关系特征, 表明研究区的湖水和河水受蒸发作用明显, 且湖水受到的蒸发作用更大; 周边地下水主 要是大气降水渗人地下形成, 而位于湖岸部分区域的井水存在异常偏正的氢氧稳定同位素值, 可能是由于 受蒸发的湖水进入该井水并与蒸发作用不明显的地下水发生了混合作用.

2) 考虑温度和湿度因子的结果显示河水的剩余水体比例在 $0.85 \sim 0.96$ 之间, 而湖水的剩余水体比例在 $0.71 \sim 0.77$ 之间, 换而言之, 河水蒸发损失的量为最初的水体的 $4 \% \sim 15 \%$, 而湖水损失量为最初的水体的 $23 \% \sim 29 \%$. 利用剩余水体中氢氧稳定同位素组成与剩余水体比例的关系计算水体剩余水体比例以及蒸发 损失的比例, 可能是评估水体受蒸发程度的一种有效的方法.

3 ) 对于缺乏实测资料的研究区, 氢氧稳定同位素平衡估算湖泊蒸发量是非常有效的方法. 利用氢氧稳 定同位素方法对呼伦湖多年平均蒸发量进行计算, 计算结果与实测值相近, 说明了该方法的可靠性. 但同时 需要指出的是, 计算过程中由于涉及的参数较多, 对蒸发量的估算结果均有不同程度的影响, 需要在计算时 考虑周全.

4) 氢氧稳定同位素对于研究区域水文过程有着重要的作用, 本文由于受制于研究区调查范围及样品采 集密度, 仅对其在上述几个方面作出了应用, 但实际上氢氧稳定同位素还有更加广泛的应用空间, 比如研究 区域地下水、湖水的补给来源, 判断承压含水层的地下水滞留时间或者地下水年龄, 降雨一径流机制等方面. 氢氧稳定同位素的应用可以加深对区域水文循环的认识, 为干旱半干旱地区水资源的利用与保护提供科学 的依据.

致谢: 感谢内蒙古农业大学河湖湿地水环境项目组同学在野外实验工作上的支持和帮助.

\section{5 参考文献}

[ 1 ] Hu HY, Bao WM, Qu SM et al. Fractionation mechanism of stable hydrogen and oxygen isotope in water body evaporating. Hydrology, 2007, 27(3) : 2-5. [胡海英, 包为民, 矍思敏等. 稳定性氢氧同位素在水体蒸发中的研究进展. 水文, $2007,27(3): 2-5$.]

[ 2 ] Shi H, Liu SR, Zhao XG. Application of stable hydrogen and oxygen isotope in water circulation. J Soil Water Conserv, 2003, 17(2) : 163-166. [石辉, 刘世荣, 赵晓广. 稳定性氢氧同位素在水分循环中的应用. 水土保持学报, 2003, 17(2) : 163-166.]

[ 3 ] Kim K, Lee XH. Isotopic enrichment of liquid water during evaporation from water surfaces. J Hydrol, 2011, 399: 364375. DOI: 10.1016/j.jhydrol.2011.01.008.

[ 4 ] Clark ID, Frjtz P. Environmental isotopes in hydrogeology. New York: Lewis Publishers, 1997.

[ 5 ] Wassenaar LI, Athanasopoulos P, Hendry MJ. Isotope hydrology of precipitation, surface and ground waters in the Okanagan Valley, British Columbia, Canada. J Hydrol, 2011, 411: 37-48. DOI: 10.1016/j.jhydrol.2011.09.032.

[ 6 ] Wang LH, Dong YH, Xie YQ et al. Distinct groundwater recharge sources and geochemical evolution of two adjacent subbasins in the lower Shule River Basin, northwest China. Hydrogeol J, 2016, 24(8) : 1967-1979. DOI: 10.1007/s10040016-1456-1.

[ 7 ] Currell MJ, Dahlhaus P, Ii H. Stable isotopes as indicators of water and salinity sources in a southeast Australian coastal wetland: identifying relict marine water, and implications for future change. Hydrogeol J, 2015, 23: 235-248. DOI: 10. 1007/s10040-014-1199-9.

[ 8 ] Cui BL, Li XY. Characteristics of stable isotope and hydrochemistry of the groundwater around Qinghai Lake, NE QinghaiTibet Plateau. China Environ Earth Sci, 2014, 71: 1159-1167. DOI: 10.1007/s12665-013-2520-y. 
[ 9 ] Tian LD, Yao TD, Sun WZ et al. Study on stable isotope fractionation during water evaporation in the middle of the Tibetan Plateau. J Glaciol Geocryol, 2000, 22(2) : 159-164. [田立德, 姚檀栋, 孙维贞等. 青藏高原中部水蒸发过程中的 氧稳定同位素变化. 冰川冻土, 2000, 22(2): 159-164.]

[10] Ma B, Liang X, Jin MG et al. Characteristics of fractionation of hydrogen and oxygen isotopes in evaporating water in the typical region of the North China Plain. Adv in Water Sci, 2015, 26(5): 639-648. [马斌, 梁杏, 靳孟贵等. 华北平原 典型区水体蒸发氢氧同位素分馏特征. 水科学进展, 2015, 26(5) : 639-648.]

[11] Qian H, Dou Y, Li XJ et al. Changes of $\delta^{18} \mathrm{O}$ and $\delta \mathrm{D}$ along Dousitu River and its indication of river water evaporation. Hydrogeol Eng Geol, 2007, 34(1) : 107-112. [ 钱会, 窦妍, 李西建等. 都思兔河氢氧稳定同位素沿流程的变化及其 对河水蒸发的指示. 水文地质工程地质, 2007, 34(1) : 107-112.]

[12] Xiao W, Zhu SX, Wang W et al. Estimating evaporation over a large and shallow lake using stable isotopic method: A case study on Lake Taihu. J Lake Sci, 2017, 29(4) : 1009-1017. DOI:10.18307/2017.0425. [肖薇, 符靖茹, 王伟等. 用稳 定同位素方法估算大型浅水湖泊蒸发量一以太湖为例. 湖泊科学, 2017, 29(4): 1009-1017.]

[13] Wu HW, Li XY, Li J et al. Evaporative enrichment of stable isotopes $\left(\delta^{18} \mathrm{O}\right.$ and $\left.\delta \mathrm{D}\right)$ in lake water and the relation to lake-level change of Lake Qinghai, Northeast Tibetan Plateau of China. J Arid Land, 2015, 7 (5) : 623-635. DOI: 10. 1007/s40333-015-0048-6.

[14] Zhang XP, Yao TD. Estimation of lake evaporation by stable isotopic ratio. J Glaciol Geocryol, 1997, 19(2) : 161-166. [章新平,姚檀栋. 利用稳定同位素比率估计湖泊的蒸发. 冰川冻土, 1997, 19(2): 161-166. ]

[15] Sun B, Li CY, Zhang S et al. Analysis of Hulun digital basin based on spatial information technology. Environ Pollut Control, 2010, 8: 5-9. [孙标, 李畅游, 张生等. 基于空间信息技术的呼伦湖流域研究. 环境污染与防治, 2010, 8: 5-9.]

[16] Yan DH, He Y, Deng W et al. Influences of eco-hydrological process to water environmental system in Hulun Lake Basin. Bull Soil Water Conserve, 2001, 21(5): 1-5. [严登华, 何岩, 邓伟等. 呼伦湖流域生态水文过程对水环境系统的影 响. 水土保持通报, 2001, 21(5): 1-5.]

[17] Craig H. Isotopic variations in meteoric waters. Science, 1961, 133: 1702-1703.

[18] Tsujimura M, Abe Y, Tanaka T et al. Stable isotopic and geochemical characteristics of groundwater in Kherlen River basin, a semi-arid region in eastern Mongolia. J Hydrol, 2007, 333: 47-57. DOI:10.1016/j.jhydrol.2006.07.026.

[19] Wang HC ed. The summary of stable isotope in hydrogeology. Beijing: Geological Publishing House, 1991. [王恒纯. 氢氧 同位素水文地质概论. 北京: 地质出版社, 1991.]

[20] Dansgaard W. Stable isotopes in precipitation. Tellus, 1964, 16(4) : 436-468.

[21] Majoube M. Oxygen-18 and deuterium fractionation between water and steam. J Chim Phys PCB, 1971, 68 (10): 1423-1436.

[22] Zhang XP, Yao TD, Tian LD. Study on the fractionation mechanism of stable isotope in evaporating water body. J Glaciol Geocryol, 2003, 25(1): 65-71. [章新平, 姚檀栋, 田立德. 水体蒸发过程中稳定同位素分馏的模拟. 冰川冻土, $2003,25(1): 65-71$.]

[23] Craig H, Gordon IJ. Isotopic exchange effects in the evaporation of water. J Geophys Res, 1963, 68: 5079-5087.

[24] Gonfiantini R. Environmental isotopes in lake studies. Terr Environ, 1986, 2 : 132-168.

[25] Saxena RK ed. Oxygen- $0^{18}$ fractionation in nature and estimation of groundwater recharge. Uppsala: Fyris-Tryck AB, 1987: 16-32.

[26] Jacob H, Sonntag C. An 8-year record of the seasonal variation of ${ }^{2} \mathrm{H}$ and ${ }^{18} \mathrm{O}$ in atmospheric water vapour and precipitation at Heidelberg. Tellus B, 1991, 43B (3) : 291-300.

[27] Seal RR. Stable isotope geochemistry of mine waters and related solids. Mineral Soc Can, 2003, (1) : 303-334.

[28] Gibson JJ, Edwards TWD, Bursey GG et al. Estimating evaporation using stable isotopes: Quantitative results and sensitivity analysis for two catchments in northern Canada. Nord Hydrol, 1993, 24: 79-94.

[29] Sun B. The Dynamic Change of water based on spatial information technology for Hulun Lake in Inner Mongolia [ Dissertation]. Hohhot: Inner Mongolia agricultural University, 2010. [孙标. 基于空间信息技术的呼伦湖水量动态演化研究 [学位论文]. 呼和浩特: 内蒙古农业大学, 2010.] 